\title{
La codificación como instrumento en la gestión clínica de las unidades del dolor
}

\author{
M.S. Acedo Gutiérrez ${ }^{1}$, S. Rodríguez Sánchez², E. González García², M.P. Sanz Ayán², A. Martínez Salio² \\ y L. Vicente Fatela ${ }^{2}$ \\ ${ }^{1}$ Servicio de Medicina Interna. Hospital Virgen de la Torre. Madrid. ${ }^{2}$ Unidad Multidisciplinar para el Estu- \\ dio y Tratamiento del Dolor. Hospital Universitario 12 de Octubre. Madrid
}

Acedo Gutiérrez MS, Rodríguez Sánchez S,González García E, Sanz Ayán MP, Martínez Salio A, Vicente Fatela L. La codificación como instrumento en la gestión clínica de las unidades del dolor. Rev Soc Esp Dolor 2015;22(4):145-158.

\begin{abstract}
Introduction: The main tools of information for clinical management are based on the systematic collection of clinical data, which is embodied in the MDS (Minimum Basic Data Set), and the use of patient classification systems, being the AP-DRGs those adopted in our country for the health administration. The data needed to perform the grouping DRGs are contained in the MDS and among them are: The main diagnosis, interventions or other procedures performed and secondary diagnoses, which should be coded with ICD-9-CM. The DRG system is a management tool that allows an analytical accounting system, through the relative weights of each DRG.

Objectives: To show the codification of diagnoses that are carried out in a Multidisciplinary Unit of Chronic Pain by ICD-9$\mathrm{CM}$, unique system officially recognized by our health services, and demonstrate the importance that correct coding of diagnoses of pain has to obtain the DRGs that best describe the degree of complexity of the processes that are treated, with the corresponding "cost per case".

Material and methods: We reviewed the medical records to obtain the information necessary to perform the encoding. For each diagnoses a master code (main diagnoses) is assigned and if necessary one or more secondary codes are added. It is assigned a code for the procedure performed. Once the codification is obtain, along with the necessary data for each
\end{abstract}

Recibido: 29-07-14

Aceptado: 15-10-14 patient, the various DRGs are obtained, with their corresponding weights.

Results: Diagnoses were coded with ICD-9-CM from 2002 until 2012, performing a database with encoding of the most common diagnoses in a chronic pain unit. It has been obtained each year information about grouping of pathologies by DRG, and its weights. From 2002 to 2009 the most common DRG was the 243, "Medical pathology of spine", that account for $57 \%$ of cases. Beginning in 2010 , the new codification was introduced, including the code 338 and as a consequence the DRGs changed. The category 338, "Pain not classified under other concepts", is part of the chapter of Diseases of the Nervous System and Senses Organs. The most frequent DRGs were: 035 "Other disorders of nervous system without CC", 42.28\% of cases; 243 "Medical pathology spine" $18 \%$ and 019 "Disorders of cranial and peripheral nerves without CC" $15.5 \%$. The characteristics of these DRGs are described together the diagnoses of pain that are included in each of them.

Conclusions: The use of coding diagnoses and procedures with ICD-9-CM is an effort to unify the language used in clinical practice. It is necessary that the coding is done correctly and consistently to get results that reflect real activity that takes place in a service and what it costs. In order for the codification to become relevant in the clinical management by processes, it is essential that the unit managers must be familiar with these tools. In the medium term, the used of ICD-10-CM will be established in clinical practice in Spain.

Key words: ICD-9-CM. DRG. Codification. Pain unit.

\section{RESUMEN}

Introducción: los principales instrumentos de información para la gestión clínica se basan en la recogida sistemática de datos clínicos, que se materializa en el CMBD, y en la utilización de sistemas de clasificación de pacientes, siendo los AP-GRD los adoptados en nuestro país por la administración sanitaria. Los datos necesarios para realizar la agrupación en GRD están 
contenidos en el CMBD y entre ellos están: diagnóstico principal, intervenciones o procedimientos y diagnósticos secundarios, que deben estar codificados con la CIE-9-MC. El sistema GRD es un instrumento de gestión, que permite una contabilidad analítica, a través de los pesos relativos de cada GRD.

Objetivos: mostrar la codificación de los diagnósticos que se realizan en una Unidad Multidisciplinar de Dolor Crónico mediante la CIE-9-MC, único sistema reconocido a nivel institucional, y evidenciar la importancia que tiene la codificación correcta de los diagnósticos de dolor para obtener los GRD que describan más adecuadamente el grado de complejidad de los procesos tratados, con su correspondiente "coste por proceso".

Material y métodos: se han revisado las historias clínicas para obtener la información necesaria para realizar la codificación. A cada diagnóstico se le asigna un código principal y si es preciso uno o varios códigos secundarios. Se debe asignar un código para el procedimiento realizado. Una vez realizada la codificación, junto con los datos necesarios de cada paciente, se obtienen los diferentes GRD, con sus pesos correspondientes.

Resultados: se han codificado diagnósticos con la CIE-9-MC desde el año 2002 al 2012, realizando una base de datos con la codificación de los diagnósticos más frecuentes en una Unidad de Dolor. Se ha obtenido cada año información sobre la agrupación de patologías mediante GRD, y su peso correspondiente. De 2002 a 2009 el GRD más frecuente fue el 243, "Patología médica de columna vertebral" en el $57 \%$ de los casos. A partir del año 2010 se introdujo la nueva codificación que incluye el código 338 y como consecuencia cambiaron los GRD. La categoría 338, "Dolor no clasificado bajo otros conceptos", se enmarca dentro del capítulo de Enfermedades del Sistema Nervioso y Órganos de los Sentidos. Los GRD más frecuentes han sido: 035, "Otros trastornos del sistema nervioso, sin CC" en el 42,28\% de los casos; 243 "Patología médica de columna vertebral" en el $18 \%$ y 019 "Trastornos de nervios craneales y periféricos sin CC" en el 15,5\%. Se describen las características de estos GRD y los diagnósticos de dolor que se han incluido en cada uno de ellos.

Conclusiones: el uso de la codificación de diagnósticos y procedimientos con la CIE-9-MC supone un esfuerzo por unificar el lenguaje que se utiliza en la práctica clínica. Es preciso que la codificación se realice correcta y consistentemente para obtener resultados que reflejen la actividad real que se realiza en un servicio y lo que cuesta. Para que realmente la codificación influya en la gestión clínica por procesos, es fundamental que los responsables de las unidades conozcan estas herramientas. A medio plazo, se instaurará en la actividad clínica en España el uso de la CIE-10-MC.

Palabras clave: CIE-9-MC. GRD. Codificación. Unidad de dolor.

\section{INTRODUCCIÓN}

El diccionario de la Real Academia de la Lengua Española define código, entre otras acepciones, como la combinación de letras, números u otros caracteres que tiene un determinado valor dentro de un sistema establecido o bien como un sistema de signos y de reglas que permite formu- lar y comprender un mensaje. La codificación es el proceso por el cual la información de una fuente es convertida en símbolos para ser comunicada. Así, la codificación clínica permite unificar y homogeneizar el lenguaje que se utiliza para definir las diferentes categorías nosológicas y los procedimientos. A través de la asignación de un código a una enfermedad, se intenta que los profesionales normalicen el lenguaje que se utiliza en la práctica clínica, es decir que "se llame a lo mismo con el mismo nombre". Las áreas de Documentación y de Sistemas de Información y Control de gestión de nuestros hospitales han creado una base de datos médicos en la que se identifican, en un lenguaje normalizado, los diagnósticos que motivaron la hospitalización del paciente, las comorbilidades presentes en el momento del ingreso, las complicaciones que pudieran presentarse y los procedimientos médicos/quirúrgicos que fueron realizados. Este lenguaje normalizado se obtiene a través de la asignación de un código, incluido en la $9^{\mathrm{a}}$ revisión de la Clasificación Internacional de Enfermedades-Modificación Clínica, basada en criterios de la Organización Mundial de la Salud (OMS).

Haciendo un poco de historia, la OMS publica la $9^{\text {a }}$ revisión de la Clasificación Internacional de Enfermedades en 1977, que tiene su origen en la "Lista Internacional de Causas de Defunción" del Instituto Internacional de Estadística de Viena, desarrollada por William Farr en 1855. Posteriormente, en 1978, se modifica, añadiéndose un eje clínico de morbilidad y un volumen de procedimientos tanto diagnósticos como terapéuticos. Pasa entonces a denominarse $9^{\mathrm{a}}$ revisión de la Clasificación Internacional de Enfermedades-Modificación Clínica (CIE-9-MC), nombre que se mantiene hasta la actualidad. Se produce así una adaptación para el uso clínico de una clasificación cuyo origen fue para causas de muerte (1). Una década más tarde, en 1988, es traducida al castellano y publicada por el INSALUD (2,3). Por otra parte, en 1987 se aprobó el Conjunto Mínimo Básico de Datos, CMBD, para aplicarlo a los informes de alta hospitalaria (4). Se define como el número mínimo de variables que permita obtener un máximo de aplicaciones clínicas y administrativas. Así, el CMBD es una base de datos clínicos y administrativos que proceden de la historia clínica y se obtienen al alta del paciente (5). El INSALUD en el año 1988 inició el proyecto CÓDIGO, cuyo objetivo era la implantación, de forma voluntaria, de la recogida de datos para cubrir el CMBD, así como la codificación con la CIE-9-MC $(4,6)$. Una historia de 25 años que ha adquirido importancia en los últimos años debido a la irrupción de la gestión clínica en los hospitales españoles. En la comunidad de Madrid se regula con el Decreto 89/1999 del BOCM, la recogida con carácter obligatorio del CMBD de alta hospitalaria y cirugía mayor ambulatoria (7).

La importancia del CMBD está determinada por la necesidad de disponer de una fuente de datos uniforme y 
suficiente, que posibilite los procesos de gestión hospitalaria, la implantación de nuevos sistemas de financiación, la elaboración de indicadores de rendimiento y utilización, el control de la calidad asistencial y la disponibilidad de información para la investigación clínica y epidemiológica. En el CMBD, la codificación clínica de diagnósticos y procedimientos se realiza con la CIE-9-MC, que se actualiza bianualmente, siendo la última edición la novena, en vigor desde enero del año 2014 (8). Las normas de codificación clínica para el Sistema Nacional de Salud se recogen en los Boletines de Codificación Clínica y están disponibles en: www.msssi.gob.es, antigua www.msc.es (5). La utilidad del CMBD es permitir la construcción de sistemas de clasificación de pacientes que permitan comparar la eficacia y calidad de los hospitales y los servicios hospitalarios. En España, el sistema de clasificación de pacientes utilizado para el análisis y elaboración de indicadores generales es el All Patient Diagnosis Related Groups (AP-GRD) $(5,9,10)$. Sin ser el único, el sistema de agrupamiento de Grupos Relacionados por el Diagnóstico (GRD) es el más universalmente aceptado. Es un sistema de clasificación de pacientes basado en la agrupación de diagnósticos más o menos afines y en la combinación de diferentes atributos predictivos del nivel de recursos hospitalarios que estos consumen. Se trata pues de un sistema de clasificación de episodios de hospitalización en base a isoconsumo de recursos (11). Los GRD fueron inicialmente desarrollados en la Universidad de Yale por R. Fetter, J. Thompson y cols. a partir de la octava revisión de la Clasificación Internacional de Enfermedades (12). En España, su uso oficial como herramienta de medición del producto hospitalario, tiene lugar a través del proyecto de "Análisis y desarrollo de los GRD en el Sistema Nacional de Salud", aprobado el 17 de noviembre de 1997 por el Ministerio de Sanidad y Consumo y las Comunidades Autónomas (13). El propósito original de los GDR era medir el rendimiento de un hospital, es decir, los pacientes que trata un hospital para facilitar una mejora de la calidad en el mismo. Los GRD utilizan variables demográficas y de diagnóstico para clasificar a los pacientes en grupos que se puedan comparar clínicamente, con duraciones de estancia en el hospital y con consumos de recursos similares. Por lo tanto, los costes de tratamiento para los casos incluidos en cada GDR deben ser similares. Bajo los AP-GRD, los casos se asignan a uno de los más de 680 grupos diferentes, que varían según la versión utilizada $(13,14)$.

Como ya se ha referido, la información que necesita el sistema GRD para clasificar a los pacientes está contenida en el CMBD. Los datos necesarios para realizar la agrupación en GRD son: edad, sexo, circunstancias del alta (si el paciente está vivo o fallecido, se ha trasladado a otro hospital o ha sido un alta voluntaria), el diagnóstico principal (motivo del ingreso), intervenciones u otros procedimientos que se han realizado al paciente durante su ingreso, diagnósticos secundarios que coexisten con el principal en el momento del ingreso o se han desarrollado durante el ingreso. Los tres últimos, diagnósticos principal y secundarios, así como intervenciones o procedimientos, deben estar codificados con la CIE-9-MC $(10,13)$.

La agrupación GRD es única y excluyente, de modo que cada paciente (en realidad cada episodio de hospitalización) se clasifica en un GRD que queda descrito por: un número, un título que pretende ser descriptivo de su contenido, una descripción de si es un GRD médico o quirúrgico y un peso que pondera el consumo de recursos necesarios para asistir ese tipo de pacientes. Al clasificar un registro del alta hospitalaria, el sistema GRD lee primero el diagnóstico principal (el que motivó el ingreso), y lo clasifica en la Categoría Diagnóstica Mayor (CDM) correspondiente. Después analiza si tiene un código de intervención quirúrgica, y si es así lo clasifica entre los códigos GRD quirúrgicos. En caso de no tener un código de intervención quirúrgica, lo clasifica entre los códigos GRD médicos que corresponda. Por último, en algunos casos, según la edad, la circunstancia del alta o eventuales diagnósticos secundarios que puede tener el paciente, lo clasifica en un GRD más específico. Así, cada CDM tiene unos GRD quirúrgicos y otros médicos. En casi todas las CDM existen GRD quirúrgicos o médicos con "complicación o comorbilidad" (con CC) o con "complicación o comorbilidad mayor" (con CCM) para agrupar a determinados pacientes que tienen diagnósticos secundarios significativos que aumentan la complejidad de cuidados (10). Dada la relevancia que adquiere la diferenciación entre diagnóstico principal y los eventuales diagnósticos secundarios para realizar la agrupación, es importante aclarar, lo que en términos de codificación se entiende por: a) diagnóstico principal: es la enfermedad que tras el estudio, y por tanto el alta, el médico que atendió al paciente establece como causa del ingreso. Por tanto, el diagnóstico principal no es necesariamente el más importante o el más grave, sino el que originó el ingreso; y b) diagnóstico secundario: son las enfermedades coexistentes con el diagnóstico principal en el momento del ingreso o que se desarrollan durante la estancia hospitalaria e influyen en su duración o en el tratamiento administrado (10).

Este sistema de GRD es un instrumento de gestión, pues permite calcular el coste de cada GRD, es decir, permite tener una contabilidad analítica, a través de los pesos relativos de cada GRD. Los pesos GRD establecen los recursos que se pueden necesitar para tratar un caso de un GRD determinado. El peso relativo de un GRD expresa una estimación del peso relativo respecto al paciente promedio de hospitalización de agudos, siendo el patrón de referencia igual a 1 (13). La CIE-9-MC ha sido actualizada tanto en los diagnósticos como en los procedimientos y, como consecuencia de ello y de los cambios de la asistencia hospitalaria en los últimos años, los GRD han sufrido cambios: apari- 
ción de nuevos grupos, desaparición de preexistentes y ajustes del peso en todos y cada uno de ellos (10). Existe así una validación continua de los GRD y una constante revisión de su escala de pesos relativos. Puede variar, por ejemplo desde 0,3108 para el GRD 351 "esterilización de varón" a 19, 2422 para el GRD 829 "trasplante de páncreas", según la norma del año 2012, publicada por el Ministerio de Sanidad, Servicios Sociales e Igualdad, de la versión 27.0 de AP-GRD, versión en vigor actualmente (15).

La gestión clínica está sustentada en tres pilares básicos: garantizar una práctica clínica apropiada, favorecer una organización centrada en las necesidades de los pacientes y en la producción de procesos clínicos, y desarrollar sistemas de información centrados en el proceso asistencial que constituyan herramientas para evaluar la calidad de la gestión clínica (16). Los principales instrumentos de información para la gestión clínica se basan en la recogida sistemática de datos clínicos, que se materializa en el CMBD, y en la utilización se sistemas de clasificación de pacientes, siendo los AP-GRD los adoptados en nuestro país por la administración sanitaria. Los GRD se han convertido en un modelo de medida del producto hospitalario, que ha facilitado la comparación entre hospitales, la gestión por líneas de producto y la evaluación de actividad, productividad y eficiencia hospitalaria. Los indicadores de los GRD al estar vinculados con los servicios responsables, secciones, áreas de gestión clínicas, el hospital, los hospitales de una comunidad autónoma, permiten comparar resultados y orientar en la mejora de las prácticas clínicas, pudiendo ser utilizados tanto para la gestión clínica, como para la contabilidad analítica. Se dispone así de un lenguaje y una terminología común y manejable para todos los estamentos directivos del hospital. Tanto médicos como administradores y gerentes pueden referir sus respectivos argumentos asistenciales, económicos o de gestión, a unos productos definidos y aceptados por el colectivo.

Este trabajo busca dos objetivos, mostrar la evolución de la codificación de los diagnósticos que se realizan en una Unidad de Dolor Crónico, mediante la CIE-9-MC, que es el sistema de clasificación de enfermedades basado en criterios de la OMS y el único reconocido en nuestro medio a nivel institucional, y evidenciar la importancia que tiene la codificación correcta de los diagnósticos de dolor crónico, para obtener los GRD que describan más adecuadamente el grado de complejidad de los procesos que se tratan en una Unidad Multidisciplinaria de Dolor Crónico, con su correspondiente "coste por proceso", instrumento necesario para la gestión clínica.

\section{MATERIAL Y MÉTODOS}

El origen de nuestro interés por la codificación de diagnósticos y procedimientos en las Unidades de Dolor Cró- nico, surge a raíz del uso en nuestro hospital de un sistema informático de recogida de actividad hospitalaria, denominado HP-HIS (Sistema Integral Hospitalario-Hewlett-Packard), donde era necesario introducir los datos de aquellos pacientes a los que se les realizaba algún procedimiento o cirugía. En el año 2002 nuestra unidad se incorporó a dicho sistema, y se observó que los códigos que se utilizaban para describir los diagnósticos de dolor, no expresaban la complejidad de nuestros pacientes, por lo que decidimos de manera activa participar en dicha codificación. Se formó un equipo en el que participamos dos facultativos y la enfermera de la Unidad, junto con una profesional cualificada de sistemas de información y control de gestión.

El sistema de clasificación de enfermedades a utilizar fue la CIE-9-MC, que es el único actualmente reconocido de forma institucional en nuestro país, como ya se ha comentado. Así, se puede disponer de un lenguaje normalizado que permita a los profesionales realizar análisis comparativos con los mismos criterios, aunque no reflejan aspectos psicopatológicos, es decir, criterios que engloben trastornos cognitivo-conductuales, medioambientales, sociales, etc. que acompañan muchas veces al dolor, ni la terminología que se utiliza actualmente en la clínica.

La CIE-9-MC permitía localizar la región anatómica afectada por el dolor, pero no aportaba información sobre las características del mismo, no describía si se trataba de un dolor agudo o crónico, si estaba relacionado con una neoplasia o con una cirugía. Para clasificar estos y otros aspectos relacionados con el dolor, la ICD9CM incorporó en el año 2006 ( $6^{\text {a }}$ edición CIE-9-MC 2008 en España) la categoría 338, "Dolor no clasificado bajo otros conceptos", incluida en el Capítulo 6 de Enfermedades del Sistema Nervioso y Órganos de los Sentidos (320-389) (17). Previo al año 2008, en la CIE-9-MC, no existía ninguna categoría específica para dolor, por lo que se tuvo que codificar cada diagnóstico revisando los códigos que mejor describían cada uno de ellos, de manera "casi artesanal" (18). A partir de 2008, con la introducción de la categoría 338, la mayoría de los diagnósticos cambiaron de código, por lo que hubo que revisar la base de datos de los diagnósticos previamente codificados.

Es necesario revisar las historias clínicas para obtener la información necesaria para poder realizar la codificación. A cada diagnóstico se le asigna un código principal (diagnóstico principal) y si es preciso uno o varios códigos secundarios (diagnósticos secundarios), para refinar más el diagnóstico. Existen unas normas básicas de codificación que se deben aplicar para codificar de una manera eficiente (18). Además, en 2010 se publicó una guía oficial ICD-9-MC para la codificación, donde se dan una serie de normas para codificar el dolor tanto agudo como crónico (19). Por otra parte, fruto del trabajo realizado en nuestra unidad durante estos 10 años, en marzo de 2012, vio su luz el boletín de codificación número 37, donde se recoge 
la "Actualización en la codificación del dolor" (20). Este documento publicado por el Ministerio de Sanidad, Servicios Sociales e Igualdad, y respaldado por la Sociedad Española del Dolor, encierra las normas actualizadas de la codificación del dolor, tanto en su vertiente diagnóstica como terapéutica, válidas para todo el territorio español. Es un boletín orientado para su uso por los codificadores, por lo que se hace una breve descripción de los diagnósticos y procedimientos de forma sencilla, para que puedan ser comprendidos por profesionales no sanitarios.

Se presentan algunos ejemplos de diagnósticos de dolor crónico codificados siguiendo estas normas (los códigos deben ir en el orden asignado):

- Ejemplo 1. Diagnóstico cuyo código es único (sólo código principal) y aparece como tal en la CIE-9-MC, buscando en el índice alfabético de enfermedades: "Meralgia parestésica".

355.1 Meralgia parestésica.

- Ejemplo 2. Diagnóstico con un código principal y otro secundario: "Dolor crónico mixto, nociceptivo somático lumbar y neuropático, con radiculopatía asociada, secundario a estenosis del canal lumbar".

338.29 Otro dolor crónico.

724.02 Estenosis lumbar, lumbosacra.

- Ejemplo 3. Diagnóstico con un código principal y dos secundarios: "Dolor crónico neuropático periférico secundario a polineuropatía diabética por diabetes mellitus tipo 2".

338.29 Otro dolor crónico.

250.60 Diabetes tipo 2 o no especificada con manifestaciones neurológicas.

357.2 Polineuropatía en diabetes.

- Ejemplo 4. Diagnóstico con un código principal y tres secundarios: "Dolor crónico nociceptivo somático localizado en muñeca secundario a fractura de Colles antigua".

338.21 Dolor crónico debido a trauma.

719.44 Dolor articular en mano.

905.2 Efecto tardío de fractura de extremidad superior. E989 Efectos tardíos de lesión, sin determinarse si fue infligido de forma accidental o intencionada.

- Ejemplo 5. Diagnóstico con un código principal y cuatro secundarios: "Dolor crónico en rodilla secundario a prótesis de rodilla dolorosa".

996.77 Otra complicación por prótesis interna de articulación.

338.28 Otro dolor crónico postoperatorio.

719.46 Dolor articular en pierna.

E878.1 Intervención quirúrgica con implante de dispositivo como causa de reacción anormal del paciente o de complicación posterior, sin mención de accidente en el momento de la intervención.

V43.65 Estado de sustitución de articulación de rodilla.
Como se ha podido observar, el grado de complejidad de la codificación depende del interés de codificar correctamente lo que se hace. Si nos conformamos con un solo código, por ejemplo el 338.29 "Otro dolor crónico", estaremos perdiendo una información muy valiosa acerca de las patologías que tratamos y en consecuencia no podremos analizar nuestros datos con rigor.

Por otra parte, además se debe asignar un código para el procedimiento realizado. La clasificación de procedimientos en la CIE-9-MC tiene dos divisiones principales: la lista tabular y el índice alfabético de procedimientos. Los códigos básicos de procedimientos tienen dos dígitos y una posición decimal. Puede ser ampliado a una segunda posición decimal para ofrecer más información sobre el lugar anatómico, procedimiento o técnica específica (18). Se aconseja consultar el boletín de codificación previamente citado donde se describen la mayoría de los procedimientos que se utilizan en el tratamiento del dolor, con su correspondiente código, debido a que en la CIE-9-MC no existe un capítulo específico para procedimientos en dolor, estando dispersos en diversos capítulos, en ocasiones difíciles de localizar. Se ha tomado como base el Catálogo de Procedimientos de las Unidades de Dolor Crónico realizado por el Panel de Expertos de la Sociedad Madrileña del Dolor y que coinciden con los incluidos en el documento editado por el Ministerio de Sanidad, Política Social e Igualdad, "Unidad de tratamiento de dolor: estándares y recomendaciones" (2022). Se describen algunos ejemplos de codificación de procedimientos (20):

- Ejemplo 1. Procedimientos no invasivos.

99.27 Iontoforesis.

93.39 Estimulación eléctrica transcutánea.

- Ejemplo 2. Bloqueos de nervios periféricos. Los bloqueos nerviosos periféricos se encuentran codificados en la subcategoría 04.8 "Inyección dentro de nervio periférico":

04.80 Inyección de nervio periférico, no especificada de otra manera.

04.81 Inyección de anestésico dentro del nervio periférico con fines analgésicos.

En este código están incluidos los siguientes tipos de bloqueos: bloqueo del ganglio de Gasser, bloqueo nervio intercostal, bloqueo del nervio trigémino, bloqueo de nervios craneales, bloqueo nervioso de plexos, bloqueo troncular, bloqueo facetario, otros bloqueos periféricos.

- Ejemplo 3. Bomba de infusión implantable.

- Implante $86.06+03.90$

86.06 Implante bomba de infusión.

03.90 Inserción de catéter en canal espinal, espacio (epidural) (subaracnoideo) (subdural) para infusión (continua o intermitente) de sustancia terapéutica o paliativa. 
- Relleno y programación:

03.91 Inyección de anestésico en el conducto espinal para analgesia.

03.92 Inyección de otro agente en canal espinal.

- Explante de BIC:

86.05 Incisión con extracción de cuerpo extraño o dispositivo de piel y tejido celular subcutáneo.

- Retirada del catéter:

03.99 Otras operaciones sobre la médula espinal y estructuras del conducto espinal.

Una vez realizada la codificación adecuada, junto con los datos necesarios de cada paciente, se obtienen los diferentes GRD, con sus pesos correspondientes. Hay que tener en cuenta que tanto los GRD como sus pesos se revisan de forma continuada, por lo que según la versión utilizada del AP-GRD, que varía según el año, el peso relativo para un GRD determinado puede cambiar.

\section{RESULTADOS}

Se han codificado diagnósticos con la CIE-9-MC desde el año 2002 hasta el año 2012, realizando una base de datos con la codificación que se ha considerado más correcta de los diagnósticos más frecuentes en una Unidad de Dolor Crónico. Como se ha comentado, a partir de la introducción del código 338, la codificación de la mayoría de los diagnósticos ha variado, teniendo que poner al día dicha base de datos. En las tablas I, II y III se describen ejemplos de codificación de diversos diagnósticos en dolor nociceptivo, dolor neuropático y dolor oncológico. La codificación de los procedimientos se describe íntegra- mente en el boletín de codificación número 37 publicado por Ministerio de Sanidad, Servicios Sociales e Igualdad, al que se remite (20).

Se ha obtenido cada año información sobre la agrupación de patologías mediante GRD, y el peso correspondiente a cada uno de ellos. Las diferentes versiones de AP-GRD según los años que se han codificado son: año 2002 a 2005 inclusive: versión (v.) 18.0; años 2006-2007: v. 21.0; años 2008-2009: v. 23.0; año 2010: v. 25.0; y años 2011-2012: v. 27.0 .

Desde 2002 a 2009 se utilizó una codificación donde no se incluía el código 338. Centrándonos en los datos del año 2008 (Tabla IV), similares a los años previos, se observa que el GRD más frecuente fue el 243, cuya descripción es "Patología médica de columna vertebral", que ha supuesto el 57\% de los casos. Otros GRD frecuentes fueron: 247 "Signos/síntomas del sistema músculo-esquelético-conectivo"; 019 "Trastornos de nervios craneales y periféricos sin CC"; 244 "Enfermedad ósea/artropatía específica con CC". Por otra parte, los GRD relacionados con sistema nervioso (GRD 12, 19, 25, 35) supusieron el $8,6 \%$ y los GRD relacionados con el sistema músculoesquelético (GRD 239, 241, 243, 244, 245, 247, 248, 256) el $84,2 \%$ de los casos.

A partir del año 2010, se introdujo la nueva codificación y como corresponde cambiaron los GRD obtenidos. Hay que tener en cuenta que la categoría 338, "Dolor no clasificado bajo otros conceptos", se enmarca dentro del capítulo de Enfermedades del Sistema Nervioso y Órganos de los Sentidos, por lo que todos los diagnósticos que se asocien a este código van a pertenecer a GRD relacionados con el sistema nervioso. Por ello, es a través de los códigos/

TABLA I. EJEMPLOS DE CODIFICACIÓN DE DOLOR CRÓNICO POR ESPONDILOARTROSIS

\begin{tabular}{|c|c|c|}
\hline Diagnóstico & Código $1 .^{\circ}$ & Código $2 .^{\circ}$ \\
\hline $\begin{array}{l}\text { Dolor crónico nociceptivo somático cervical } \pm \text { neuropático, con radiculopatía asociada, } \\
2 .^{\circ} \text { a espondiloartrosis cervical/cervicodorsal }\end{array}$ & 338.29 & 721.0 \\
\hline $\begin{array}{l}\text { Dolor crónico nociceptivo somático dorsal } \pm \text { neuropático, con radiculopatía asociada, } \\
2 .^{\circ} \text { a espondiloartrosis torácica/dorsal }\end{array}$ & 338.29 & 721.2 \\
\hline $\begin{array}{l}\text { Dolor crónico nociceptivo somático lumbar } \pm \text { neuropático, con radiculopatía asociada, } \\
2 .^{\circ} \text { a espondiloartrosis lumbar/lumbosacra/sacra }\end{array}$ & 338.29 & 721.3 \\
\hline $\begin{array}{l}\text { Dolor crónico nociceptivo somático } \pm \text { neuropático, con radiculopatía asociada, } \\
2 .^{\circ} \text { a espondiloartrosis sin especificar }\end{array}$ & 338.29 & 721.90 \\
\hline $\begin{array}{l}\text { Dolor crónico mixto nociceptivo somático cervical y neuropático con mielopatía, } \\
2^{\circ} \text { a espondiloartrosis cervical/cervicodorsal }\end{array}$ & 338.29 & 721.1 \\
\hline $\begin{array}{l}\text { Dolor crónico mixto nociceptivo somático torácico y neuropático con mielopatía, } \\
\text { 2. }^{\circ} \text { a espondiloartrosis torácica/dorsal }\end{array}$ & 338.29 & 721.41 \\
\hline $\begin{array}{l}\text { Dolor crónico mixto nociceptivo somático lumbar/sacro y neuropático con mielopatía, } \\
2 .^{\circ} \text { a espondiloartrosis lumbar/lumbosacra/sacra }\end{array}$ & 338.29 & 721.42 \\
\hline
\end{tabular}


TABLA II. EJEMPLOS DE CODIFICACIÓN DE DOLOR NEUROPÁTICO

\begin{tabular}{|c|c|c|c|}
\hline Diagnóstico: Dolor crónico neuropático & $\begin{array}{c}\text { Código } \\
\text { principal }\end{array}$ & Código 2 & Código 3 \\
\hline Dolor crónico neuropático central, secundario a siringomielia & 338.0 & 336.0 & \\
\hline Dolor crónico neuropático central, secundario a accidente cerebro-vascular & 338.0 & 438.89 & \\
\hline $\begin{array}{l}\text { Dolor crónico neuropático periférico } 2 .^{\circ} \text { a neuropatía de nervio mediano } \\
\text { (síndrome del túnel del carpo) sin intervenir }\end{array}$ & 354.0 & & \\
\hline $\begin{array}{l}\text { Dolor crónico neuropático periférico } 2 .^{\circ} \text { a neuropatía de nervio mediano } \\
\text { (síndrome del túnel del carpo) intervenido }\end{array}$ & 338.28 & 354.0 & \\
\hline Dolor crónico neuropático: Síndrome del miembro fantasma doloroso & 353.6 & & \\
\hline Dolor crónico neuropático: SDRC tipo I postraumático en MMSS & 337.21 & & \\
\hline Dolor crónico neuropático: SDRC tipo I postraumático en MMII & 337.22 & & \\
\hline Dolor crónico neuropático: SDRC tipo I no postraumático & 733.7 & & \\
\hline Dolor crónico neuropático: SDRC tipo II en MMSS, $2^{\circ}$ a cualquier diagnóstico & 354.4 & & \\
\hline Dolor crónico neuropático: SDRC tipo II en MMII, 2. a a cualquier diagnóstico & 355.71 & & \\
\hline Dolor crónico neuropático periférico $2 .^{\circ}$ a polineuropatía urémica & 338.29 & 585 & 357.4 \\
\hline Dolor crónico neuropático periférico $2 .^{\circ}$ a polineuropatía alcohólica & 338.29 & 357.5 & \\
\hline Dolor crónico neuropático periférico $2 .^{\circ}$ a polineuropatía diabética si DM 2 & 338.29 & 250.60 & 357.2 \\
\hline Dolor crónico neuropático periférico $2 .^{\circ}$ a polineuropatía diabética si DM 1 & 338.29 & 250.61 & 357.2 \\
\hline
\end{tabular}

SDRC: síndrome de dolor regional complejo; MMII: miembros inferiores; MMSS: miembros superiores; DM: diabetes mellitus.

TABLA III. EJEMPLOS DE CODIFICACIÓN DE DOLOR ONCOLÓGICO

\begin{tabular}{|c|c|c|c|}
\hline Diagnóstico: Dolor oncológico & $\begin{array}{l}\text { Código } \\
\text { principal }\end{array}$ & Código 2 & Código 3 \\
\hline Dolor crónico oncológico por mieloma múltiple (sin remisión) & 338.3 & 203.00 & \\
\hline Dolor crónico oncológico por mieloma múltiple (recidiva) & 338.3 & 203.01 & \\
\hline $\begin{array}{l}\text { Dolor crónico oncológico } 2 .^{\circ} \text { a metástasis óseas de adenoCa. de próstata }(\mathrm{Ca} \\
\text { próstata presente) }\end{array}$ & 338.3 & 198.5 & 185 \\
\hline $\begin{array}{l}\text { Dolor crónico oncológico } 2 .^{\circ} \text { a metástasis óseas de adenoCa. de próstata } \\
\text { (prostatectomía) }\end{array}$ & 338.3 & 198.5 & V45.77 \\
\hline Dolor crónico oncológico $2 .^{\circ}$ a metástasis óseas de Ca mama & 338.3 & 198.5 & 174.8 \\
\hline Dolor crónico oncológico $2 .^{\circ}$ a metástasis óseas de Ca de pulmón & 338.3 & 198.5 & 162.9 \\
\hline Dolor crónico oncológico por Ca pleural o mesotelioma & 338.3 & 786.52 & 163.9 \\
\hline Dolor crónico oncológico por Ca de páncreas & 338.3 & 157.8 & \\
\hline Dolor crónico oncológico por Ca hepático primario & 338.3 & 155.0 & \\
\hline Dolor crónico oncológico por Ca hepático secundario & 338.3 & 197.7 & \\
\hline
\end{tabular}

Ca: cáncer.

diagnósticos secundarios como se va a obtener la información sobre la patología que está produciendo ese cuadro de dolor crónico. A continuación se analizan los datos resultantes en el año 2011, con la adaptación de los nuevos códigos diagnósticos (Tabla V). Los GRD más frecuentes han sido: 035, cuya descripción es "Otros trastornos del sistema nervioso, sin CC", suponiendo el 42,28\% de los casos (635/1502); 243 "Patología médica de columna vertebral" 
TABLA IV. DESCRIPCIÓN DE GRD DEL AÑO 2008 (AP-GRD 23.0)

\begin{tabular}{clcc}
\hline GRD & \multicolumn{1}{c}{ Descripción GRD } & Peso GRD & Casos \\
\hline 012 & Trastornos degenerativos del sistema nervioso & 1,5147 & 15 \\
019 & Trastornos de los nervios periféricos y craneales, sin cc & 0,9449 & 128 \\
025 & Convulsiones y cefalea, edad $\geq 18$ sin cc & 0,8083 & 6 \\
035 & Otros trastornos del sistema nervioso, sin cc & 0,9274 & 19 \\
239 & Fract. patológica/n. maligna s. músculo-esquelético-conectivo & 2,0335 & 51 \\
241 & Enfermedades del tejido conectivo, sin cc & 0,9282 & 11 \\
243 & Patología médica de columna vertebral & 0,7919 & 1.117 \\
244 & Enfermedades óseas y artropatía específica, con cc & 1,1917 & 119 \\
245 & Enfermedades óseas y artropatía específica, sin cc & 0,7055 & 67 \\
247 & Signos y síntomas s. musculoesquelético y tejido conectivo & 0,5989 & 168 \\
248 & Tendinitis, miositis y bursitis & 0,7029 & 35 \\
256 & Otros diagnósticos s. musculoesquelético y tejido conectivo & 0,7989 & 82 \\
284 & Enfermedades menores de la piel, sin cc & 0,5761 & 71 \\
301 & Alteraciones endocrinas, sin cc & 0,7589 & 9 \\
352 & Otros diagnósticos del aparato reproductor masculino & 0,4417 & 6 \\
414 & Otros trastornos mieloproliferativos mal diferenciados sin cc & 1,62 & 1 \\
464 & Signos y síntomas sin cc & 0,6131 & 55 \\
& & 0,8317 & 1.960 \\
\hline
\end{tabular}

cc: complicación o comorbilidad; frac: fractura; n: neoplasia; s: sistema.

el 18\% (272/1502); y 019 "Trastornos de nervios craneales y periféricos sin CC" el 15,5\% (233/1502).

El GRD 035, "Otros trastornos del sistema nervioso central sin CC", es un GRD médico que agrupa a pacientes ingresados por una enfermedad del sistema nervioso que no han sido clasificados en otro GRD más específico. Los motivos de ingreso más frecuentes en estos pacientes son diagnósticos como: mielopatía o compresión de médula espinal, lesiones cerebrales anóxicas, trastornos de la sensibilidad, trastornos de la marcha, neurofibromatosis, ciertas apneas centrales primarias u orgánicas, trastornos del ritmo circadiano del sueño, distonía o discinesia secundaria a fármacos, dolor central o crónico, narcolepsia y trastornos miotónicos. También se incluyen los pacientes que ingresaron para implantación o ajuste de una derivación ventricular cerebral o un estimulador cerebral, espinal o de nervio periférico. El GRD 034 "Otros trastornos del sistema nervioso central con CC" tiene la misma descripción, añadiendo que además estos pacientes tienen otro diagnóstico etiquetado de complicación o comorbilidad como: metástasis cerebral, espinal o de pulmón, enfermedad pulmonar obstructiva crónica, infección urinaria o insuficiencia renal (10). Como se observa dentro de estos GRD se incluyen patologías dispares, varias de ellas no relacionadas con el dolor. Además, las complicaciones o comorbilidades asociadas hace que un paciente pase de un GRD a otro, en este caso del GRD 035 al 034, variando su peso, de 0,8847 a 1,357, con lo que ello supone una diferente financiación. En la tabla VI se presentan los diagnósticos de dolor que se han incluido en estos GRD. Se observa que los diagnósticos principales en el GRD 034, también están incluidos en el GRD 035. Entonces, ¿cuál ha sido el dato diferencial que ha provocado que un determinado episodio clínico se haya asociado a uno u otro GRD? Podría haber sido la presencia de una de las comorbilidades que se han referido previamente, pero en nuestro caso no se codificó ninguna patología asociada que no tuviera relación con el dolor, es decir, que lo que se hizo fue codificar el diagnóstico según se ha descrito en los apartados previos, utilizando varios códigos. En la tabla VII se exponen algunos ejemplos que explican estas diferencias. Así para el diagnóstico o código principal 338.0 "Síndrome de dolor central", hay dos diagnósticos que en la historia clínica aparecerían como: "Dolor crónico neuropático central secundario a siringomielia" (338.0 + 336.0) y "Dolor crónico neuropático central secundario a accidente cerebro-vascular" (338.0 + 438.89). La diferen- 
TABLA V. DESCRIPCIÓN DE GRD DEL AÑO 2011 (AP-GRD 27.0)

\begin{tabular}{clcc}
\hline GRD & \multicolumn{1}{c}{ Descripción GRD } & Casos & Peso medio \\
\hline 012 & Trastornos degenerativos del sistema nervioso & 4 & 1,4782 \\
019 & Trastornos de los nervios periféricos y craneales, sin cc & 233 & 0,9196 \\
034 & Otros trastornos del sistema nervioso, con cc & 63 & 1,3157 \\
035 & Otros trastornos del sistema nervioso, sin cc & 635 & 0,8847 \\
140 & Angor pectoris & 9 & 0,8209 \\
241 & Enfermedades del tejido conectivo, sin cc & 13 & 0,9093 \\
243 & Patología médica de columna vertebral & 272 & 0,7853 \\
244 & Enfermedades óseas y artropatía específica, con cc & 1 & 1,1057 \\
245 & Enfermedades óseas y artropatía específica, sin cc & 13 & 0,7146 \\
247 & Signos y síntomas s. musculoesquelético y tejido conectivo & 70 & 0,5986 \\
248 & Tendinitis, miositis y bursitis & 33 & 0,7132 \\
249 & Cuidados post s. musculoesquelético y tejido conectivo & 7 & 1,1721 \\
256 & Otros diagnósticos s. musculoesquelético y tejido conectivo & 44 & 0,7792 \\
284 & Enfermedades menores de la piel, sin cc & 15 & 0,5651 \\
294 & Diabetes, edad $\geq 36$ & 1 & 0,9317 \\
301 & Alteraciones endocrinas, sin cc & 9 & 0,718 \\
352 & Otros diagnósticos del aparato reproductor masculino & 7 & 0,4719 \\
463 & Signos y síntomas con cc & 53 & 1,2225 \\
464 & Signos y síntomas sin cc & 20 & 0,6138 \\
& & 1.502 & 0,8737 \\
\hline
\end{tabular}

CC: complicación o comorbilidad; s: sistema.

cia entre uno y otro es el diagnóstico o código secundario, que ha conducido al GRD asignado, con una diferencia en el peso medio importante, que varía según la versión APGRD que se utilice.

El GRD 019, "Trastornos de nervios craneales y periféricos sin CC", es un GRD médico que agrupa a pacientes ingresados por una enfermedad de nervios craneales y periféricos de origen vírico, metabólico, traumático o idiopático como: neuropatía diabética, neuralgia del trigémino, parálisis de Bell, síndrome del túnel carpiano, síndrome cervicobraquial, neuropatía periférica idiopática, o lesiones de los nervios cubital, radial, ciático o plexo braquial. El GRD 018, "Trastornos de nervios craneales y periféricos con CC", tiene la misma descripción añadiéndose además que estos pacientes tienen otro diagnóstico etiquetado de complicación o comorbilidad como: enfermedad pulmonar obstructiva crónica, úlcera crónica en pierna o infección urinaria (10). Los diagnósticos de dolor crónico que se han obtenido en el GRD 019 se describen en la tabla VIII.

También se quiere hacer referencia a los GRD 463 "Signos y síntomas con CC" y 464 "Signos y síntomas sin CC".
Ambos son GRD médicos que agrupan a pacientes ingresados por signos o síntomas diversos como: dolor post-operatorio, ascitis, edema o caquexia, sin que se haya llegado a un diagnóstico más específico como motivo de ingreso. La diferencia es que el GRD 463 requiere que además estos pacientes tengan otro diagnóstico etiquetado de complicación o comorbilidad como: derrame pleural, insuficiencia renal o enfermedad pulmonar obstructiva crónica (10). Los diagnósticos de dolor oncológico son incluidos en estos GRD, y vienen codificados con el 338.3, descrito como "Dolor (agudo) (crónico) relacionado con una neoplasia".

Por último, como resulta obvio de la exposición previa, según el código diagnóstico que se elija para una determinada patología varía el GRD y por tanto su peso relativo. Así, por ejemplo, para un cuadro de dolor crónico nociceptivo localizado en región lumbar secundario a una espondiloartrosis, se pueden utilizar diferentes denominaciones: a) lumbalgia (código 724.2) que correspondería al GRD 243, cuyo peso en AP-GRD versión 25.0 es 0,7853; b) lumboartrosis o espondiloartrosis lumbar (721.3), también GRD 243 y mismo peso 0,7853 ; y c) dolor crónico 
TABLA VI. GRD 35 “OTROS TRASTORNOS DEL SISTEMA NERVIOSO, SIN CC” Y GRD 034 “OTROS TRASTORNOS DEL SISTEMA NERVIOSO, CON CC”

\begin{tabular}{cclc}
\hline GRD (peso) & Código principal & \multicolumn{1}{c}{ Descripción diagnóstico principal } & $N^{{ }^{o}}$ \\
\hline $035(0,8847)$ & 338.0 & Síndrome de dolor central & 1 \\
& 338.21 & Dolor crónico debido a trauma & 19 \\
& 338.22 & Dolor crónico postoracotomía & 17 \\
& 338.28 & Dolor crónico postoperatorio & 47 \\
& 338.29 & Otro dolor crónico & 533 \\
& 338.4 & Síndrome de dolor crónico & 2 \\
& 348.1 & Lesiones cerebrales anóxicas & 7 \\
Total 035 & 359.9 & Miopatía no especificada & 9 \\
\hline GRD (peso) & Código principal & & 635 \\
\hline $034(1,3157)$ & 338.0 & Síndrome de dolor central & $N .^{{ }^{*}}$ \\
& 338.21 & Dolor crónico debido a trauma & 16 \\
& 338.22 & Dolor crónico postoracotomía & 1 \\
Total 034 & 338.29 & Otro dolor crónico & 21 \\
\hline
\end{tabular}

CC: complicación o comorbilidad.

TABLA VII. SÍNDROME DE DOLOR CENTRAL (338.0). DOLOR CRÓNICO POSTORACOTOMÍA (338.22). DOLOR CRÓNICO DEBIDO A TRAUMATISMO (338.21)

\begin{tabular}{|c|c|c|c|c|c|}
\hline $\begin{array}{c}\text { Cód. } \\
\text { principal }\end{array}$ & Cód. $2^{\circ}$ & Diagnóstico principal & $G R D$ & Descripción GRD & Peso \\
\hline 338.0 & {$[336.0]$} & Síndrome de dolor central & 34 & Otros trastornos del $\mathrm{SN}$ con $\mathrm{CC}$ & $\begin{array}{l}\text { v. } 25.01,3157 \\
\text { v. } 27.01,18\end{array}$ \\
\hline 338.0 & [438.89] & Síndrome de dolor central & 35 & Otros trastornos del $\mathrm{SN} \sin \mathrm{CC}$ & $\begin{array}{l}\text { v. } 25.00,8847 \\
\text { v. } 27.00,81\end{array}$ \\
\hline 338.22 & [162.9] & Dolor crónico postoracotomía & 34 & Otros trastornos del $\mathrm{SN}$ con $\mathrm{CC}$ & $\begin{array}{l}\text { v. } 25.01,3157 \\
\text { v. } 27.01,18\end{array}$ \\
\hline 338.22 & [354.9] & Dolor crónico postoracotomía & 35 & Otros trastornos del $\mathrm{SN}$ sin $\mathrm{CC}$ & $\begin{array}{l}\text { v. } 25.00,8847 \\
\text { v. } 27.00,81\end{array}$ \\
\hline 338.22 & & Dolor crónico postoracotomía & 35 & Otros trastornos del $\mathrm{SN} \sin \mathrm{CC}$ & $\begin{array}{l}\text { v. } 25.00,8847 \\
\text { v. } 27.00,81\end{array}$ \\
\hline 338.21 & [721.3] & $\begin{array}{l}\text { Dolor crónico debido a } \\
\text { traumatismo }\end{array}$ & 35 & Otros trastornos del $\mathrm{SN} \sin \mathrm{CC}$ & $\begin{array}{l}\text { v. } 25.00,8847 \\
\text { v. } 27.00,81\end{array}$ \\
\hline 338.21 & {$[805.4]$} & $\begin{array}{l}\text { Dolor crónico debido a } \\
\text { traumatismo }\end{array}$ & 34 & Otros trastornos del SN con $\mathrm{CC}$ & $\begin{array}{l}\text { v. } 25.01,3157 \\
\text { v. } 27.01,18\end{array}$ \\
\hline 338.21 & & $\begin{array}{l}\text { Dolor crónico debido a } \\
\text { traumatismo }\end{array}$ & 35 & Otros trastornos del $\mathrm{SN}$ sin $\mathrm{CC}$ & $\begin{array}{l}\text { v. } 25.00,8847 \\
\text { v. } 27.00,81\end{array}$ \\
\hline
\end{tabular}

Cód: código; SN: sistema nervioso; CC: complicación o comorbilidad; v: versión AP-GRD.

336.0: Siringomielia. 438.89: Otro efecto tardío de enfermedad cerebrovascular. 162.9: Neoplasia maligna de bronquios y pulmón, parte no especificada. 354.9: Mononeuritis de miembro superior no especificada. 721.3: Espondilosis lumbosacra sin mielopatía. 805.4: Fractura de columna vertebral lumbar cerrada. 
TABLA VIII. GRD 019 “TRASTORNOS DE LOS NERVIOS PERIFÉRICOS Y CRANEALES, SIN CC”

\begin{tabular}{cclc}
\hline GRD (peso) & Código principal & \multicolumn{1}{c}{ Descripción diagnóstico principal } & $N^{{ }^{o}}$ \\
\hline $019(0,9196)$ & 053.12 & Neuralgia trigémino post-herpética & 2 \\
& 053.13 & Polineuropatía post-herpética & 18 \\
250.60 & Diabetes con manifestaciones neurológicas tipo 2 o no especificada & 15 \\
337.21 & Distrofia simpático refleja de miembro superior (SDRC tipo I) & 58 \\
& 337.22 & Distrofia simpático refleja de miembro inferior (SDRC tipo I) & 55 \\
& 337.29 & Distrofia simpático refleja de otro sitio especificado (SDRC tipo I) & 13 \\
& 337.9 & Alteración del sistema nervioso autónomo no especificada & 5 \\
& 353.6 & Síndrome de miembro fantasma & 14 \\
& 353.8 & Otros trastornos de raíces y plexos nerviosos & 8 \\
Total 019 & 354.4 & Causalgia (SDRC tipo II) & 42 \\
& 355.1 & Meralgia parestésica & 1 \\
& 355.9 & Mononeuritis de sitio no especificado & 1 \\
\hline
\end{tabular}

CC: complicación o comorbilidad; SDRC: síndrome de dolor regional complejo.

por lumboartrosis (códigos 338.29+721.3) con GRD 035, cuyo peso es 0,8847 . Por otra parte, según el término que se utilice, tendremos no sólo información sobre la presencia de dolor sino también sobre la etiología de dicho dolor. El término lumbalgia sólo indica dolor a nivel lumbar pero no su causa y si es agudo o crónico. El término lumboartrosis indica la patología causante del dolor, pero en términos de codificación el dolor no aparece por ningún sitio. En cambio si utilizamos para la codificación el diagnóstico de "Dolor crónico por lumboartrosis", aparecerá un código de dolor y otro de la patología causante. Así, en el ejemplo, el peso es mayor para el GRD 035 y teniendo en cuenta que esta patología es una de las más prevalentes en las unidades de dolor crónico, aparte de perder información sobre el diagnóstico, estamos perdiendo financiación si utilizamos los otros códigos.

\section{DISCUSIÓN}

John Bonica comprendió la necesidad de un lenguaje común entre los distintos especialistas que trabajan con pacientes con dolor desde el principio. Esta idea la refleja Derasari en un editorial de la revista Pain (2002), donde describía la situación de la medicina del dolor como "una torre de Babel" y resaltaba la idea de MersKey y Bogduk, sobre la importancia de la taxonomía para los clínicos, destacando los beneficios de una clasificación eficaz de los síndromes de dolor $(23,24)$. La clasificación del dolor crónico desarrollada por la IASP es la más detallada entre las propuestas hasta hoy en día. Se trata de una clasificación multiaxial, con base teórica, de los síndromes de dolor crónico, diseñada para estandarizar las descripciones de los síndromes dolorosos y poder codificarlos. El subcomité de taxonomía realizó la primera clasificación en 1986 y la IASP publicó en 1994 la versión revisada, con una clasificación del dolor crónico que incluye una taxonomía completa, así como las diferentes definiciones; ambas son objeto de revisiones y actualizaciones periódicas. Consta de cinco ejes, que se centran sobre todo en las manifestaciones físicas del dolor, e incluye: Eje I: región anatómica. Eje II: sistema orgánico. Eje III: características temporales. Eje IV: valoración por el paciente de la intensidad y el momento de comienzo. Eje V: Etiología. Permite comentarios de los factores psicológicos en dos ejes: el eje II, en que se puede codificar un trastorno mental y el eje $\mathrm{V}$ en el cual los posibles factores etiológicos pueden ser psicofisiológicos y psicológicos (23-25). Así, se dispone de una clasificación que describe con exactitud los síndromes de dolor en términos útiles para el diagnóstico y relevantes para el tratamiento, además de contemplar el dolor con sus implicaciones psicosociales. A pesar de que esta clasificación pudiera ser la más óptima, en el ámbito europeo se utiliza el sistema de clasificación de enfermedades basado en criterios de la OMS, la CIE-9-MC, que es el único reconocido en nuestro medio a nivel institucional y con el que se realiza la codificación "oficial". El uso de la codificación de diagnósticos y procedimientos con la CIE-9-MC 
supone un esfuerzo por unificar y hacer más homogéneo el lenguaje que se utiliza en la práctica clínica. Es preciso que la codificación se realice correcta y consistentemente para obtener resultados que reflejen la actividad real que se lleva a cabo en un servicio y lo que cuesta.

Es importante tener una formación básica sobre el proceso de codificación. Si bien no es necesario llegar a un nivel de implicación como el que surgió en nuestra unidad, sí es fundamental que se implique a los profesionales de Sistemas de Información y Control de Gestión de nuestros hospitales, que vayan a codificar nuestra actividad. Para ello, debemos tener claro de qué estamos hablando y trasmitir con buenos argumentos la importancia de una codificación lo más correcta posible. Es esencial un esfuerzo conjunto entre clínicos y codificadores para lograr una documentación, codificación y recuperación de la información completa y precisa. Nunca se insistirá demasiado en la importancia de una documentación consistente y completa en la historia clínica. Sin esta documentación es una tarea difícil, sino imposible, aplicar cualquier norma de codificación (26). Nuestra labor es dejar reflejado en los informes de forma correcta y teniendo en cuenta lo que se quiere codificar, la mejor descripción del diagnóstico principal y los secundarios, así como los procedimientos. Según se ha reflejado, dado el diferente peso de un GRD debido a las comorbilidades asociadas, se ve conveniente que si un paciente tiene patología no relacionada con dolor, como enfermedad pulmonar obstructiva crónica, infección urinaria, insuficiencia renal o úlcera crónica en pierna, esta sea reflejada como diagnóstico secundario. Así facilitaremos la labor a los profesionales que se encargan de la codificación, y ello conducirá a una mejora segura de nuestra actividad. Es importante que la información se recoja con la máxima fiabilidad y exactitud, por lo que es necesario estar al día de las actualizaciones periódicas, no sólo de la CIE-9-MC, sino también de las publicaciones monográficas en forma de Boletines de Codificación, que realiza el Ministerio de Sanidad, Servicios Sociales e Igualdad.

Para que realmente la codificación influya en la gestión clínica por procesos, es fundamental que los responsables de las unidades conozcan estas herramientas, así podrían disponer de un contrato-programa que recoja el presupuesto clínico asignado en relación a los medios diagnósticos y terapéuticos con los que cuenta y la actividad que se realiza. La utilización del sistema de codificación representa una mejora en la gestión de las unidades favoreciendo los estudios clínicos, epidemiológicos, de investigación y la financiación por procesos. En los tiempos actuales, la gestión se impone; no debemos dejarla de lado, pero sí debemos "humanizarla". No se trata de obtener números, se trata de que esos números describan lo más fehacientemente posible nuestro trabajo diario y que eso repercuta en un mejor cuidado de nuestros pacientes.
A medio plazo, se instaurará en la actividad clínica en España el uso de la CIE-10-MC. La CIE-10 fue publicada en 1992. La OMS autorizó al National Center for Health Statistics (NCHS) para desarrollar una modificación clínica de CIE-10, a la que se denomina CIE-10-MC y está destinada a sustituir a la anterior modificación clínica, la CIE9-MC, que se ha estado utilizando desde los años 70. En la CIE-10-MC, el dolor está ubicado de nuevo en el capítulo 6: "Enfermedades del sistema nervioso y órganos de los sentidos", representando la categoría G89 "Dolor, no clasificado en otro lugar" (26). Las normas de codificación de diagnósticos para dolor son similares a las de la CIE-9-MC, por lo que posiblemente no sea difícil actualizar los códigos de la CIE-9-MC, con la CIE-10-MC. Pero existe una diferencia fundamental: la CIE-9-MC contiene una clasificación de procedimientos, la CIE-10-MC no la incluye. Se ha desarrollado un sistema independiente de codificación de procedimientos de la $10^{a}$ revisión de la Clasificación Internacional de Enfermedades, la CIE-10-PCS (Procedure Coding System). La CIE-10-PCS ha sido creada para acompañar a la clasificación de diagnósticos CIE-10-MC. Este nuevo sistema de codificación de procedimientos se desarrolló para sustituir a los códigos de procedimientos de la CIE-9-MC en pacientes hospitalizados. A diferencia de la CIE-9-MC, la CIE-10-PCS fue diseñada para hacer posible que cada código tuviese una estructura estandarizada y muy descriptiva, siendo no obstante lo suficientemente flexible como para dejar espacio a futuras ampliaciones, con la aparición de nuevas técnicas. Todos los códigos de CIE-10-PCS tienen una longitud de siete caracteres. Cada una de las posiciones del código representa un aspecto del procedimiento: posición 1: sección, 2: sistema orgánico, 3: tipo de procedimiento, 4: localización anatómica, 5: abordaje, 6: dispositivo y 7: calificador. A cada posición de un código se le puede asignar uno de los 34 posibles valores: los números del 0 al 9 y cualquiera de los caracteres alfabéticos (excepto la I y la $\mathrm{O}$, dado que pueden confundirse fácilmente con el 1 y el 0). El significado de cada caracter depende de la posición que ocupa en el código, un mismo valor colocado en diferentes posiciones del código, significa cosas diferentes. La CIE-10-PCS presenta diferencias fundamentales con la CIE-9-MC, en cuanto a estructura, organización y posibilidades. Presenta una estructura multiaxial del código, que hace posible que sea exhaustiva, susceptible de expansión y que proporcione un alto grado de flexibilidad y funcionalidad. En la CIE-9-MC hay procedimientos realizados sobre localizaciones anatómicas distintas y utilizando diferentes abordajes o dispositivos que pueden tener el mismo código de procedimiento. En la CIE-10-PCS se puede construir un código único para cada procedimiento significativamente distinto. Así mismo, ha sido diseñada para actualizarse fácilmente a medida que se necesiten nuevos códigos para nuevos procedimientos y nuevas técnicas. No se incluye información diagnóstica 
en ninguno de los códigos de CIE-10-PCS. Son los códigos diagnósticos y no los de procedimiento los que deben especificar la razón por la que se realiza la técnica (27).

Se presenta un futuro de cambios en cuanto a la codificación de procedimientos, que promete poder expresar con códigos mucho más específicos los procedimientos que se utilizan en el tratamiento del dolor, que ahora nos parecen escasos y poco descriptivos. Será necesario profundizar en la CIE-10-PCS, para que con un mayor conocimiento, se inicie la revisión de la codificación de las técnicas y procedimientos utilizados de forma habitual en las unidades de dolor. La actualización nunca termina.

\section{CORRESPONDENCIA:}

M. ${ }^{a}$ Soledad Acedo Gutiérrez

Servicio de Medicina Interna

Hospital Virgen de la Torre

c/ Puerto de Lumbreras, 5

28031 Madrid

e-mail: marisolacedo@hotmail.com

\section{BIBLIOGRAFÍA}

1. Israel RA. The history of the International Classification of Dissease. Health Bulletin 1991;49:62-6.

2. Unidad de Garantía de Calidad Total, Ministerio de Sanidad y Consumo. Manual de uso de la Clasificación Internacional de Enfermedades-9 $9^{\text {a }}$ Revisión- Modificación Clínica. Madrid: Instituto Nacional de la Salud; 1990.p.9-10.

3. Clasificación Internacional de Enfermedades. 9a revisión. Modificación Clínica. Madrid: Ministerio de Sanidad y Consumo. Secretaria Técnica; 1988.

4. Contenido del CMBD. Consejo Interterritorial del Sistema Nacional de Salud (CISNS) 14.12.1987.

5. Conjunto Mínimo Básico de Datos [en línea]. Madrid: Dirección General de Sistemas de Información Sanitaria Servicio Madrileño de Salud [consulta 28/7/14]. Disponible en: http://cmbd.sanidadmadrid.org/

6. Recogida del CMBD. Norma interna de la Dirección General INSALUD. 24.1.1992.

7. Boletín Oficial de la Comunidad de Madrid. Decreto 89/1999, de 10 de junio, por el que se regula el conjunto mínimo básico de datos (CMBD) al alta hospitalaria y cirugía ambulatoria, en la Comunidad de Madrid. BOCM, 22 de junio de 1999, núm 146, p. 10-13.

8. Clasificación Internacional de Enfermedades. 9a revisión. Modificación clínica, $9^{a}$ ed. Madrid: Ministerio de Sanidad, Servicios Sociales e Igualdad. Secretaria General Técnica; 2014 [consulta 28/7/14]. Disponible en: www.msssi.gob.es/ estadEstudios/estadisticas/docs/CIE9MC_2014_def_accesible.pdf

9. All Patient Diagnosis Related Groups (AP-DRGs). Definitions Manual. Versión 21. Wallingford: eM Health Information Systems; 2003.
10. Yetaro Laguna J, López Arbeloa C. Manual de descripción de los grupos relacionados por el diagnóstico. AP-GRD versión 25.0. $5^{\text {a }}$ ed. Vitoria-Gasteiz: Osakidetza, Servicio vasco de Salud; 2010.

11. Casas M, Guasch E. GRD e información para la gestión. En: Casas M, editor. Los grupos relacionados con el diagnóstico, experiencias y perspectivas de su utilización. Barcelona: Masson y SG; 1991. p. 179-219.

12. Fetter RB, Shin Y, Freeman JL, Averil RF, Thompson JD. "Case mix" definition by Diagnosis Related Groups. Med Care 1980;18(Supl. 2):1-53.

13. Proyecto de análisis y desarrollo de los Grupos Relacionados por el Diagnóstico (GDR) en el Sistema Nacional de Salud [en línea]. Madrid: Centro de publicaciones, Ministerio de Sanidad y Consumo, 1997 [consulta: 28/7/14]. Disponible en la web: msssi.gob.es/estadEstudios/estadísticas/ docs/análisis.pdf.

14. Subdirección General de Información Sanitaria e Innovación. Atención Ambulatoria Especializada: pesos y costes procedimientos ambulatorios en SNS (publicación en internet). Madrid. Ministerio de sanidad, Servicios Sociales e Igualdad; 2013 [consulta: 28/7/14]. Disponible en: msssi. gob.es/estadEstudios/estadísticas/inforRecopilaciones/anaDesarrolloGRD.htm.

15. Norma año 2012 AP27-Total SNS [en línea]. Madrid: Ministerio de Sanidad, Servicio Sociales e Igualdad [consulta: 28/7/14]. Disponible en: msssi.gob.es/estadEstudios/ estadísticas/cmbd.htm

16. Pérez Mateos C, Prieto I y Keller I. La acreditación hospitalaria: un reto para la sanidad española. Med Clin (Barc) 1997;109:467-74.

17. Clasificación Internacional de Enfermedades. $9^{a}$ revisión. Modificación clínica. $6^{\mathrm{a}}$ ed. Madrid: Ministerio de Sanidad y Política social. Secretaria General Técnica; 2008.

18. Vicente Fatela L, Acedo Gutiérrez MS, Rodríguez Sánchez S, González García E, Martínez Salio A, Sanz Ayan MP. Codificación del dolor crónico con la clasificación internacional de enfermedades - $9^{\mathrm{a}}$ revisión -modificación clínica (CIE-9-MC). Rev Soc Esp Dolor 2007;2:95-103.

19. ICD-9-CM Official Guidelines for Coding and Reporting. Effective October 1, 2010. Disponible en: www.cdc.gov/ nchs/data/icd9/icdguide10.pdf.

20. Acedo Gutiérrez MS ,et al. Codificación Clínica con la CIE-9-MC. Actualización en la codificación del dolor y de los procedimientos en columna, Boletín n ${ }^{\circ}$ 37, marzo 2012. Madrid: Ministerio de Sanidad, Servicios Sociales e Igualdad, 2012. Disponible en: http://www.msssi.gob. es/estadEstudios/estadisticas/normalizacion/clasifEnferm/ boletines/home.htm

21. De la Calle JL, Abejón D, Cid J, Del Pozo C, Insausti J, López E y el Panel de expertos de la Sociedad Madrileña del Dolor. Estándares de calidad asistencial y catálogo de procedimientos de las unidades de dolor crónico. Rev Soc Esp Dolor 2010;17(2):114-33.

22. Palanca Sánchez I (director), Puig Riera de Conías MM (coordinador científico), Elola Somoza J (director), Bernal Sobrino JL (comité de redacción), Paniagua Caparrós JL (comité de redacción), Grupo de Expertos. Unidad de tratamiento de dolor: estándares y recomendaciones. Madrid: Ministerio de Sanidad, Política Social e Igualdad; 2011.

23. Merskey H. Pain terms: A list with definitions and notes on usage. Recommended by IASP Subcomittee on Taxonomy. Pain 1979;6:249-52. 
24. MersKey H, Bogduk N, editores. Classification of Chronic Pain: Description of chronic pain syndromes and definitions of pain terms. Seattle: IASP Press; 1994.

25. Derasari MD. Taxonomía de los síndromes dolorosos: clasificación de los síndromes de dolor crónico. En: Prithvi Raj $\mathrm{P}$, editor. Tratamiento práctico del dolor. Madrid: EDIDE SL; 2002. p. 10-16.

26. Normativa oficial para la codificación con CIE-10-MC. Traducción de la normativa americana para ICD10MC correspondiente al año fiscal 2013 [en línea]. Madrid: Ministerio de Sanidad, Servicios Sociales e Igualdad. Subdirección general de Información Sanitaria e Innovación [consulta: 28/7/14]. Disponible en: http://www.msssi.gob.es/estadEstudios/estadisticas/docs/Norm_of_CIE10MC_2013_ V2.pdf.

27. Manual de referencia CIE 10 PCS. Traducción de la normativa americana para ICD10PCS correspondiente al año fiscal 2013 [en línea]. Madrid: Ministerio de Sanidad, Servicios Sociales e Igualdad. Subdirección general de Información Sanitaria e Innovación [consulta: 28/7/14]. Disponible en: http://www.msssi.gob.es/estadEstudios/estadisticas/docs/ CIE_10_PCS_M_Referencis_2013.pdf 